\begin{tabular}{|c|c|c|}
\hline & Journal Homepage: - www.journalijar.com & $\begin{array}{l}\text { INTERNATIONAL JOURNAL OF } \\
\text { ADVANCED RESEARCH (IJAR) }\end{array}$ \\
\hline ). $2320-5407$ & $\begin{array}{l}\text { Article DOI:10.21474/IJAR01/2452 } \\
\text { DOI URL: http://dx.doi.org/10.21474/IJAR01/2452 }\end{array}$ & \\
\hline
\end{tabular}

RESEARCH ARTICLE

\title{
ENVIRONMENTAL CONSCIOUSNESS IN PRODUCT PURCHASES - A STUDY OF STUDENTS
} COMMUNITY.

\section{Dr. Gaurav Sehgal}

Associate Professor (on Deputation) Department of HRM \& OB School of Business Studies Central University of Jammu Jammu, J\&K State India.

\section{Manuscript Info}

-........................

\section{Manuscript History}

Received: $x x x x x x x x x x x x x x x x$ Final Accepted: $x x x x x x x x x x x$ Published: $\operatorname{xxxxxxxxxxxxxxxx}$

Key words:-

Environmental Management, Green Marketing, Social Responsibility,

Product Purchase, Ethical Consumption

\section{Abstract}

Social responsibility in marketing covers a diverse range of issues, such as, green consumerism, environmentalism and social marketing. The present study focus upon environmental concern which is one of the related issues gaining prominence in the present time. It is a result of global warming that recognizes the two fold connection between environment and human society. Moreover, the G-8 currently taking about two broad parameters which are likely to become the basis of undertaking emission cuts by all countries. Firstly, overall emissions must be reduced by 50\% from current levels by the year 2050 . Secondly, the world must limit the global warming up to 2 degrees from pre-industrial level. Even an overall $50 \%$ reduction in global emission by 2050 will require each person in the developed world to cut his/her carbon footprint by over $70 \%$ over the next four decades. This will be possible only if the developed world actually embarks on a radically new consumption path. At a moral level, which is also ecologically compatible, each individual will have to examine his/her footprint and each country its development model for any meaningful consensus to evolve on the climate change debate. Though, it is difficult, yet not impossible to mitigate climate crisis.

The presented study is an attempt to study eight environmental conscious dimensions among the student community. The total sample size has been restricted to 175 students, which include a combination of rural as well as urban students. The samples have been collected from the students of two universities in the State of Jammu \& Kashmir, one each from rural area and urban area, viz-a-viz, University of Jammu, Jammu and Baba Ghulam Shah Badshah University, Rajouri. The data has been collected using two modes of data collection, viz, email and personal interaction in the university campuses. A detailed analysis was then conducted using IBM SPSS 19.0 as regard to gender and residential status (rural / urban) for testing the established hypothesis.

Copy Right, IJAR, 2016,. All rights reserved.
Corresponding Author:- Dr. Gaurav Sehgal.

Address:-Associate Professor (on Deputation) Department of HRM \& OB School of Business Studies Central University of Jammu Jammu, J\&K State India. 


\section{Introduction:-}

Consumption is good for society that it is environmentally sustainable and socially fair and tends to improve the quality of life. Some households and business firms fulfill part of their ecological responsibility by limiting family size and installing recycling and reprocessing equipment. Unless compelled by law or social custom, resource conservation practices may not become as effective or universal as the new world situation. Two things are needed, one a new attitude towards the meaning of consumption and second social organizations to implement such an attitude.

Universities play a vital role in the above stated attitude change. Universities are social organizations which deal and interact with the young minds and souls of any nation. Youth as is well said are creators of the future, henceforth, for our study the students' community from university setup was considered as the population.

Although the concept under study originated from a criticism of the consumer society, it is important to mention that the debate on related consumption with ethical position is very recent. The idea of 'ethical consumption' should initially be based on the definition of 'ethics'. In general, it refers to consumption that not only considers our personal welfare but also considers general welfare. This is a consumption in which production is completed, generating an impact on the eco-system and society in general. Under this idea, the choice of type of consumption can benefit or hinder the creation or maintenance of the productive jobs in a specific society, the preservation of ecosystem and the promotion of the general welfare of communities, countries and the entire earth. In ethical consumption, ethics as a set of values based on responsibility to oneself, to others and to the environment being the central point of all our actions in life. It includes, therefore, respect for the limits of nature and man himself, acknowledgement of the mother earth as a source of life, co-responsibility for society and the acknowledgement of the rights of others to access the resources and products that are necessary for a dignified quality of life. Ethical consumption is a growing phenomenon. An increasing number of people make their consumption decisions on the basis of ethical values, such as environmental friendly products and production methods that comply labor standards and human rights. Consumers around the globe are still much more familiar with the functional attributes of products that they are with their social attributes.

We basically need to be responsible consumers so that one buy the appropriate, right things one need. As well one should not harm environment in general and the surroundings in particular. Irresponsible buying can harm the environment. Waste is one of the kind of things that affects our entire globe, and since now people probably know that we need a healthy environment to keep us healthy and cheerful. Packaging also affects the environment to a larger extent. Some people who buy products with packaging, but most of the times these products hardly require ant packaging.

Thus, as consumers we use and create waste. Student community are the largest consumers and also larger producers of waste. Hence a control on the use of buying non-environmental friendly products by the students in social organizations such as university setup, would ideally lead to a long commitment of responsible consumerism.

\section{Hypothesis:-}

For the present study, NULL hypothesis has been established, which asserts that there is no significant difference in the static sample and population parameter under consideration. Following hypothesis have been framed to direct the analysis objectively.

$\mathbf{H}_{\mathbf{n} 1}$ : Environmental consciousness does notvary with Gender.

$\mathbf{H}_{\mathrm{n} 2}$ : Environmental consciousness does notvary with Residential Status.

\section{Sample Population:-}

Students from two universities one each in rural location and urban location were selected for the study under reference. Moreover, one of the university, viz, BGSB Universiy, Rajouri was chosen because of the reason that it is only nine years old university and caters typically to the students from minority community as well as it is located in a rural location and the second university, viz, University of Jammu was chosen because it is located in the heart of the city and is a well established university and mostly caters to the students belonging to urban community.

\section{Sample Size and Data Collection Method:-}

A total of 175 students were contacted for collecting data for the study under reference. Data was collected from the students of the two universities by two means. Some students were contacted through email, which were collected 
from personal sources as well as Placement Brochures and were asked to mark their responses on the sent word document. Also some students were made to fill up the responses for the study personally within the campus of the two universities.

\section{Dimensions / Parameters of the Study:-}

A total of eight dimensions were selected and made available for the students to mark their responses. The eight selected dimensions were arranges systematically and were required to be rated on a 5-point Likert Scale, as given below.

\begin{tabular}{|c|c|c|c|c|c|}
\hline Dimension & $\begin{array}{c}\text { Strongly } \\
\text { Agree }\end{array}$ & Agree & Neutral & Disagree & $\begin{array}{c}\text { Strongly } \\
\text { Disagree }\end{array}$ \\
\cline { 2 - 7 } & 1 & 2 & 3 & 4 & 5 \\
\hline
\end{tabular}

Recycling reduce pollution

Recycling save natural resources

Packaging should be done with recycled material

Excessive packaging damages the environment

One should refuse to buy from companied which produce environment pollution

One should avoid buying products from irresponsible companies

Read labels for their damage to the environment

One should prefer buying products in refilling containers

Theses dimensions have been adopted from one of the studies conducted by 'Brown and Wahlers' in the year 1998. The above mentioned eight dimensions were presented before the respondents in the form of questionnaire and their responses were recorded. As far as their Demographic details are concerned, for the present study only the Gender and the Residential Status (rural / urban) of the respondents were recorded.

Analysis and Observation tables:-

Table 1:- below presents the environmental consciousness in product purchase of the respondents as per their Gender.

\begin{tabular}{|l|l|r|r|r|r|}
\hline Group Statistics & Gender & $\mathrm{N}$ & Mean & $\begin{array}{c}\text { Std. } \\
\text { Deviation }\end{array}$ & $\begin{array}{c}\text { Std. Error } \\
\text { Mean }\end{array}$ \\
\hline \multirow{2}{*}{ Recycling Reduce Pollution } & Male & 82 & 1.57 & .703 & .078 \\
\cline { 2 - 6 } & Female & 93 & 1.72 & .901 & .093 \\
\hline \multirow{2}{*}{ Recycling save natural resources } & Male & 82 & 2.51 & .707 & .078 \\
\cline { 2 - 6 } & Female & 93 & 2.55 & .759 & .079 \\
\hline \multirow{2}{*}{$\begin{array}{l}\text { Packaging to be done in recycled } \\
\text { material }\end{array}$} & Male & 82 & 2.54 & 1.188 & .131 \\
\cline { 2 - 6 } $\begin{array}{l}\text { Excessive packaging damages } \\
\text { environment }\end{array}$ & Female & 93 & 2.72 & 1.386 & .144 \\
\hline \multirow{2}{*}{ Refuse to buy from accused retailer } & Male & 82 & 2.61 & 1.293 & .143 \\
\cline { 2 - 6 } & Female & 93 & 2.57 & 1.378 & .143 \\
\hline \multirow{2}{*}{$\begin{array}{l}\text { Avoid buying from irresponsible } \\
\text { company }\end{array}$} & Male & 82 & 2.63 & 1.319 & .146 \\
\cline { 2 - 6 } & Female & 93 & 2.53 & 1.119 & .116 \\
\hline \multirow{2}{*}{$\begin{array}{l}\text { Read labels for verifying damage } \\
\text { level to environment }\end{array}$} & Male & 82 & 2.88 & 1.221 & .135 \\
\cline { 2 - 6 } Buying in refillable containers & Female & 93 & 2.80 & 1.256 & .130 \\
\cline { 2 - 6 } & Male & 82 & 2.34 & 1.068 & .118 \\
\cline { 2 - 6 } & Female & 93 & 2.47 & 1.099 & .114 \\
\cline { 2 - 6 } & Male & 82 & 2.43 & 1.257 & .139 \\
\hline
\end{tabular}


Table 2:- below presents the t-test values for environmental consciousness in product purchase of the respondents as per their Gender.

\begin{tabular}{|c|c|c|c|c|c|c|c|c|c|c|}
\hline \multicolumn{11}{|c|}{ Independent Samples Test } \\
\hline & & \multicolumn{2}{|c|}{$\begin{array}{l}\text { Levene's Test } \\
\text { for Equality of } \\
\text { Variances }\end{array}$} & \multicolumn{7}{|c|}{ t-test for Equality of Means } \\
\hline & & \multirow[t]{2}{*}{$\mathrm{F}$} & \multirow[t]{2}{*}{ Sig. } & \multirow[t]{2}{*}{$\mathrm{t}$} & \multirow[t]{2}{*}{ df } & \multirow[t]{2}{*}{$\begin{array}{l}\text { Sig. } \\
(2- \\
\text { tailed })\end{array}$} & \multirow[t]{2}{*}{$\begin{array}{l}\text { Mean } \\
\text { Differe } \\
\text { nce }\end{array}$} & \multirow[t]{2}{*}{$\begin{array}{l}\text { Std. Error } \\
\text { Difference }\end{array}$} & \multicolumn{2}{|c|}{$\begin{array}{l}\text { 95\% Confidence } \\
\text { Interval of the } \\
\text { Difference }\end{array}$} \\
\hline & & & & & & & & & Lower & Upper \\
\hline \multirow{2}{*}{$\begin{array}{l}\text { Recycling } \\
\text { Reduce } \\
\text { Pollution }\end{array}$} & $\begin{array}{l}\text { Equal variances } \\
\text { assumed }\end{array}$ & 5.263 & .023 & -1.193 & 173 & .234 & -.147 & .123 & -.391 & .096 \\
\hline & $\begin{array}{l}\text { Equal variances } \\
\text { not assumed }\end{array}$ & & & -1.212 & 170.556 & .227 & -.147 & .122 & -.387 & .093 \\
\hline \multirow[t]{2}{*}{$\begin{array}{l}\text { Recycling } \\
\text { save natural } \\
\text { resources }\end{array}$} & $\begin{array}{l}\text { Equal } \\
\text { variances } \\
\text { assumed }\end{array}$ & .526 & .469 & -.325 & 173 & .746 & -.036 & .111 & -.256 & .184 \\
\hline & $\begin{array}{l}\text { Equal } \\
\text { variances not } \\
\text { assumed }\end{array}$ & & & -.326 & $\begin{array}{r}172.47 \\
1\end{array}$ & .745 & -.036 & .111 & -.255 & .183 \\
\hline \multirow{2}{*}{$\begin{array}{l}\text { Packaging to } \\
\text { be done in } \\
\text { recycled } \\
\text { material }\end{array}$} & $\begin{array}{l}\text { Equal } \\
\text { variances } \\
\text { assumed }\end{array}$ & $\begin{array}{r}3.45 \\
4\end{array}$ & .065 & -.936 & 173 & .351 & -.184 & .197 & -.572 & .204 \\
\hline & $\begin{array}{l}\text { Equal } \\
\text { variances not } \\
\text { assumed }\end{array}$ & & & -.945 & $\begin{array}{r}172.87 \\
3\end{array}$ & .346 & -.184 & .195 & -.568 & .200 \\
\hline \multirow{2}{*}{$\begin{array}{l}\text { Excessive } \\
\text { packaging } \\
\text { damages } \\
\text { environment }\end{array}$} & $\begin{array}{l}\text { Equal } \\
\text { variances } \\
\text { assumed }\end{array}$ & .207 & .650 & .196 & 173 & .844 & .040 & .203 & -.361 & .440 \\
\hline & $\begin{array}{l}\text { Equal } \\
\text { variances not } \\
\text { assumed }\end{array}$ & & & .197 & $\begin{array}{r}172.31 \\
5\end{array}$ & .844 & .040 & .202 & -.359 & .439 \\
\hline \multirow[t]{2}{*}{$\begin{array}{l}\text { Refuse to buy } \\
\text { from accused } \\
\text { retailer }\end{array}$} & $\begin{array}{l}\text { Equal } \\
\text { variances } \\
\text { assumed }\end{array}$ & $\begin{array}{r}5.08 \\
0\end{array}$ & .025 & .582 & 173 & .561 & .107 & .184 & -.257 & .471 \\
\hline & $\begin{array}{l}\text { Equal } \\
\text { variances not } \\
\text { assumed }\end{array}$ & & & .576 & $\begin{array}{r}159.73 \\
9\end{array}$ & .566 & .107 & .186 & -.261 & .475 \\
\hline \multirow{2}{*}{$\begin{array}{l}\text { Avoid buying } \\
\text { from } \\
\text { irresponsible } \\
\text { company }\end{array}$} & $\begin{array}{l}\text { Equal } \\
\text { variances } \\
\text { assumed }\end{array}$ & .281 & .597 & .438 & 173 & .662 & .082 & .188 & -.288 & .453 \\
\hline & $\begin{array}{l}\text { Equal } \\
\text { variances not } \\
\text { assumed }\end{array}$ & & & .439 & $\begin{array}{r}171.33 \\
5\end{array}$ & .661 & .082 & .187 & -.288 & .452 \\
\hline \multirow{2}{*}{$\begin{array}{l}\text { Read labels } \\
\text { for verifying } \\
\text { damage level } \\
\text { to } \\
\text { environment }\end{array}$} & $\begin{array}{l}\text { Equal } \\
\text { variances } \\
\text { assumed }\end{array}$ & .060 & .806 & -.801 & 173 & .424 & -.132 & .164 & -.456 & .193 \\
\hline & $\begin{array}{l}\text { Equal } \\
\text { variances not } \\
\text { assumed }\end{array}$ & & & -.803 & $\begin{array}{r}171.35 \\
2\end{array}$ & .423 & -.132 & .164 & -.455 & .192 \\
\hline $\begin{array}{l}\text { Buying in } \\
\text { refillable }\end{array}$ & $\begin{array}{l}\text { Equal } \\
\text { variances }\end{array}$ & .797 & .373 & .041 & 173 & .968 & .007 & .184 & $\begin{array}{l}-.355 \\
727 \\
\end{array}$ & .370 \\
\hline
\end{tabular}




\begin{tabular}{|l|l|l|l|r|r|r|r|r|r|r|}
\hline containers & assumed & & & & & & & & & \\
\cline { 2 - 8 } & $\begin{array}{l}\text { Equal } \\
\text { variances not } \\
\text { assumed }\end{array}$ & & & .040 & $\begin{array}{r}166.63 \\
9\end{array}$ & .968 & .007 & .185 & -.357 & .372 \\
\hline
\end{tabular}

Table 3:- below presents the environmental consciousness in product purchase of the respondents as per their Residential Status.

\begin{tabular}{|c|c|c|c|c|c|}
\hline \multicolumn{6}{|c|}{ Group Statistics } \\
\hline & $\begin{array}{l}\text { Residential } \\
\text { Status }\end{array}$ & $\mathrm{N}$ & Mean & Std. Deviation & $\begin{array}{l}\text { Std. Error } \\
\text { Mean }\end{array}$ \\
\hline \multirow[t]{2}{*}{ Recycling Reduce Pollution } & Urban & 81 & 1.64 & .677 & .075 \\
\hline & Rural & 94 & 1.66 & .922 & .095 \\
\hline \multirow{2}{*}{$\begin{array}{l}\text { Recycling save natural } \\
\text { resources }\end{array}$} & Urban & 81 & 2.60 & .769 & .085 \\
\hline & Rural & 94 & 2.47 & .699 & .072 \\
\hline \multirow{2}{*}{$\begin{array}{l}\text { Packaging to be done in } \\
\text { recycled material }\end{array}$} & Urban & 81 & 2.54 & 1.304 & .145 \\
\hline & Rural & 94 & 2.71 & 1.292 & .133 \\
\hline \multirow{2}{*}{$\begin{array}{l}\text { Excessive packaging damages } \\
\text { environment }\end{array}$} & Urban & 81 & 2.47 & 1.285 & .143 \\
\hline & Rural & 94 & 2.69 & 1.376 & .142 \\
\hline \multirow{2}{*}{$\begin{array}{l}\text { Refuse to buy from accused } \\
\text { retailer }\end{array}$} & Urban & 81 & 2.57 & 1.264 & .140 \\
\hline & Rural & 94 & 2.59 & 1.177 & .121 \\
\hline \multirow{2}{*}{$\begin{array}{l}\text { Avoid buying from } \\
\text { irresponsible company }\end{array}$} & Urban & 81 & 2.90 & 1.231 & .137 \\
\hline & Rural & 94 & 2.78 & 1.246 & .128 \\
\hline \multirow{2}{*}{$\begin{array}{l}\text { Read labels for verifying } \\
\text { damage level to environment }\end{array}$} & Urban & 81 & 2.57 & 1.128 & .125 \\
\hline & Rural & 94 & 2.28 & 1.031 & .106 \\
\hline \multirow[t]{2}{*}{ Buying in refillable containers } & Urban & 81 & 2.51 & 1.226 & .136 \\
\hline & Rural & 94 & 2.35 & 1.198 & .124 \\
\hline
\end{tabular}

Table 4:- below presents the t-test values for environmental consciousness in product purchase of the respondents as per their Residential Status.

\begin{tabular}{|c|c|c|c|c|c|c|c|c|c|c|}
\hline \multicolumn{10}{|c|}{ Independent Samples Test } & \\
\hline & & \multicolumn{2}{|c|}{$\begin{array}{c}\text { Levene's Test } \\
\text { for Equality of } \\
\text { Variances }\end{array}$} & \multicolumn{7}{|c|}{ t-test for Equality of Means } \\
\hline & & \multirow[t]{2}{*}{$\mathrm{F}$} & \multirow[t]{2}{*}{ Sig. } & \multirow[t]{2}{*}{$\mathrm{t}$} & \multirow[t]{2}{*}{ df } & \multirow{2}{*}{$\begin{array}{c}\text { Sig. } \\
(2- \\
\text { tailed } \\
)\end{array}$} & \multirow[t]{2}{*}{$\begin{array}{l}\text { Mean } \\
\text { Diffe } \\
\text { rence }\end{array}$} & \multirow{2}{*}{$\begin{array}{l}\text { Std. } \\
\text { Error } \\
\text { Diffe } \\
\text { rence }\end{array}$} & \multicolumn{2}{|c|}{$\begin{array}{l}\text { 95\% Confidence } \\
\text { Interval of the } \\
\text { Difference }\end{array}$} \\
\hline & & & & & & & & & Lower & Upper \\
\hline \multirow[t]{2}{*}{$\begin{array}{l}\text { Recycling } \\
\text { Reduce } \\
\text { Pollution }\end{array}$} & $\begin{array}{l}\text { Equal } \\
\text { variances } \\
\text { assumed }\end{array}$ & 3.367 & .068 & -.142 & 173 & .887 & -.018 & .124 & -.262 & .227 \\
\hline & $\begin{array}{l}\text { Equal } \\
\text { variances not } \\
\text { assumed }\end{array}$ & & & -.145 & 168.866 & .885 & -.018 & .121 & -.257 & .222 \\
\hline \multirow[t]{2}{*}{$\begin{array}{l}\text { Recycling } \\
\text { save natural } \\
\text { resources }\end{array}$} & $\begin{array}{l}\text { Equal } \\
\text { variances } \\
\text { assumed }\end{array}$ & .681 & .411 & 1.233 & 173 & .219 & .137 & .111 & -.082 & .356 \\
\hline & $\begin{array}{l}\text { Equal } \\
\text { variances not } \\
\text { assumed }\end{array}$ & & & 1.224 & 163.206 & .223 & .137 & .112 & -.084 & .358 \\
\hline \multirow{2}{*}{$\begin{array}{l}\text { Packaging } \\
\text { to be done } \\
\text { in recycled } \\
\text { material }\end{array}$} & $\begin{array}{l}\text { Equal } \\
\text { variances } \\
\text { assumed }\end{array}$ & .008 & .929 & -.862 & 173 & .390 & -.170 & .197 & -.558 & .219 \\
\hline & Equal & & & -.861 & 168.719 & .390 & -.170 & .197 & -.558 & .219 \\
\hline
\end{tabular}




\begin{tabular}{|c|c|c|c|c|c|c|c|c|c|c|}
\hline & $\begin{array}{l}\text { variances not } \\
\text { assumed }\end{array}$ & & & & & & & & & \\
\hline \multirow{2}{*}{$\begin{array}{l}\text { Excessive } \\
\text { packaging } \\
\text { damages } \\
\text { environment }\end{array}$} & $\begin{array}{l}\text { Equal } \\
\text { variances } \\
\text { assumed }\end{array}$ & .474 & .492 & 1.099 & 173 & .273 & -.222 & .202 & -.622 & .177 \\
\hline & $\begin{array}{l}\text { Equal } \\
\text { variances not } \\
\text { assumed }\end{array}$ & & & 1.104 & 171.852 & .271 & -.222 & .201 & -.620 & .175 \\
\hline \multirow{2}{*}{$\begin{array}{l}\text { Refuse to } \\
\text { buy from } \\
\text { accused } \\
\text { retailer }\end{array}$} & $\begin{array}{l}\text { Equal } \\
\text { variances } \\
\text { assumed }\end{array}$ & .709 & .401 & -.093 & 173 & .926 & -.017 & .185 & -.382 & .347 \\
\hline & $\begin{array}{l}\text { Equal } \\
\text { variances not } \\
\text { assumed }\end{array}$ & & & -.093 & 164.960 & .926 & -.017 & .186 & -.384 & .349 \\
\hline \multirow{2}{*}{$\begin{array}{l}\text { Avoid } \\
\text { buying from } \\
\text { irresponsibl } \\
\text { e company }\end{array}$} & $\begin{array}{l}\text { Equal } \\
\text { variances } \\
\text { assumed }\end{array}$ & .326 & .569 & .664 & 173 & .508 & .125 & .188 & -.246 & .495 \\
\hline & $\begin{array}{l}\text { Equal } \\
\text { variances not } \\
\text { assumed }\end{array}$ & & & .664 & 169.769 & .507 & .125 & .188 & -.246 & .495 \\
\hline \multirow{2}{*}{$\begin{array}{l}\text { Read labels } \\
\text { for verifying } \\
\text { damage } \\
\text { level to } \\
\text { environment }\end{array}$} & $\begin{array}{l}\text { Equal } \\
\text { variances } \\
\text { assumed }\end{array}$ & 1.521 & .219 & 1.784 & 173 & .076 & .291 & .163 & -.031 & .614 \\
\hline & $\begin{array}{l}\text { Equal } \\
\text { variances not } \\
\text { assumed }\end{array}$ & & & 1.772 & 163.627 & .078 & .291 & .164 & -.033 & .616 \\
\hline \multirow[t]{2}{*}{$\begin{array}{l}\text { Buying in } \\
\text { refillable } \\
\text { containers }\end{array}$} & $\begin{array}{l}\text { Equal } \\
\text { variances } \\
\text { assumed }\end{array}$ & .022 & .881 & .845 & 173 & .399 & .155 & .184 & -.207 & .517 \\
\hline & $\begin{array}{l}\text { Equal } \\
\text { variances not } \\
\text { assumed }\end{array}$ & & & .843 & 167.969 & .400 & .155 & .184 & -.208 & .518 \\
\hline
\end{tabular}

Table 5:- below presents the ANOVA table with dependent variables (the dimensions of the study) and factored with GENDER

\begin{tabular}{|c|c|c|c|c|c|c|}
\hline \multicolumn{7}{|c|}{ ANOVA } \\
\hline & & $\begin{array}{l}\text { Sum of } \\
\text { Squares }\end{array}$ & $\mathrm{df}$ & $\begin{array}{l}\text { Mean } \\
\text { Square }\end{array}$ & $\mathrm{F}$ & Sig. \\
\hline \multirow[t]{3}{*}{ Recycling Reduce Pollution } & Between Groups & .945 & 1 & .945 & 1.424 & .234 \\
\hline & Within Groups & 114.792 & 173 & .664 & & \\
\hline & Total & 115.737 & 174 & & & \\
\hline \multirow{3}{*}{$\begin{array}{l}\text { Recycling save natural } \\
\text { resources }\end{array}$} & Between Groups & .057 & 1 & .057 & .106 & .746 \\
\hline & Within Groups & 93.520 & 173 & .541 & & \\
\hline & Total & 93.577 & 174 & & & \\
\hline \multirow{3}{*}{$\begin{array}{l}\text { Packaging to be done in } \\
\text { recycled material }\end{array}$} & Between Groups & 1.473 & 1 & 1.473 & .875 & .351 \\
\hline & Within Groups & 291.121 & 173 & 1.683 & & \\
\hline & Total & 292.594 & 174 & & & \\
\hline \multirow{3}{*}{$\begin{array}{l}\text { Excessive packaging damages } \\
\text { environment }\end{array}$} & Between Groups & .069 & 1 & .069 & .039 & .844 \\
\hline & Within Groups & 310.308 & 173 & 1.794 & & \\
\hline & Total & 310.377 & 174 & & & \\
\hline \multirow{3}{*}{$\begin{array}{l}\text { Refuse to buy from accused } \\
\text { retailer }\end{array}$} & Between Groups & .501 & 1 & .501 & .339 & .561 \\
\hline & Within Groups & 256.207 & 173 & 1.481 & & \\
\hline & Total & 256.709 & 174 & & & \\
\hline Avoid buying from & Between Groups & .296 & 1 & .296 & .192 & .662 \\
\hline
\end{tabular}




\begin{tabular}{|l|l|r|r|r|r|r|}
\hline \multirow{2}{*}{ irresponsible company } & Within Groups & 265.899 & 173 & 1.537 & & \\
\cline { 2 - 7 } & Total & 266.194 & 174 & & & \\
\hline \multirow{3}{*}{$\begin{array}{l}\text { Read labels for verifying } \\
\text { damage level to environment }\end{array}$} & Between Groups & .755 & 1 & .755 & .642 & .424 \\
\cline { 2 - 7 } & Within Groups & 203.622 & 173 & 1.177 & & \\
\cline { 2 - 7 } & Total & 204.377 & 174 & & & \\
\hline \multirow{3}{*}{ Buying in refillable containers } & Between Groups & .002 & 1 & .002 & .002 & .968 \\
\cline { 2 - 7 } & Within Groups & 254.706 & 173 & 1.472 & & \\
\cline { 2 - 7 } & Total & 254.709 & 174 & & & \\
\hline
\end{tabular}

\section{Interpretations:-}

Table-1- presents the mean, standard deviation and the standard error for the data on the basis of Gender. The last column of the table represents the measure of dispersion around the mean. In a normal distribution, $68 \%$ of cases fall within one standard deviation of the mean and $95 \%$ of cases fall within two standard deviations. Thus this column of the observation table helps us to determine as to how many cases at $95 \%$ would lie between the specified no's in a normal distribution. From the table we observe that, there exists a significant difference in opinion among the males and females as regard to the eight dimensions under study. The following dimensions shows the major variation in opinion among Gender study: Recycling reduces population; Packaging to be done in recycled material; Excessive packaging damages environment; Refuse to buy from accused retailer and Buying in refillable containers.

Table-2- presents the Independent Samples Test values. The first column after the dimensions column of the table labeled as ' $\mathrm{F}$ ' tests if the variances are equal. When the $\mathrm{F}$ value is large and the significance level is small (smaller than say $0.10,0.05$, or 0.01 ) the hypothesis of equal variances can be rejected. The next column labeled 'Sig.' is also known as 'p-value' and represents the conditional probability that a relationship as strong as the one observed in the data would be present, if the null hypothesis were true. Typically a value of less than 0.05 is considered significant. From the table we observe that, for the dimensions at S.No. 1, 2, 3, 5 and 8 the hypothesis of equal variances shall not be considered while for the dimensions at S.No. 4, 6 and 7 we shall ignore the values corresponding to equal variances not assumed.

Furthermore, while looking at the column of Sig. we observe that the Null Hypothesis stated for the study, viz, Environmental consciousness does notvary with Gender stands REJECTED.

Table-3- presents the mean, standard deviation and the standard error for the data on the basis of Residential Status. From the table we observe that, there does not exists a significant difference in opinion among the respondents coming from urban and rural statas, as regard to the eight dimensions under study.

Table-4- presents the Independent Samples Test values. From the table we observe that, for the dimensions at S.No. 1and 2 the hypothesis of equal variances shall not be considered while for the dimensions at S.No. 3, 4, 5, 6, 7 and 8 we shall ignore the values corresponding to equal variances not assumed.

Furthermore, while looking at the column of Sig. we observe that the Null Hypothesis stated for the study, viz, Environmental consciousness does notvary with Residential Status stands ACCEPTED.

Table-5- presents the ANOVA test that has been conducted to find out whether there is any significant difference in the Mean value of environmental consciousness while purchasing product across different categories of respondents.

The second last column of the table labeled as 'F' tests the ratio of two mean squares. When the F value is large and the significance level is small (typically smaller than 0.05 or 0.01 ) the null hypothesis can be rejected. In other words, a small significance level indicates that the results probably are not due to random chance. The next column labeled 'Sig.' is also known as 'p-value' and represents the conditional probability that a relationship as strong as the one observed in the data would be present, if the null hypothesis were true. Typically a value of less than 0.05 is considered significant. From the table we observe that, the following dimensions do not present a significant difference in the mean value of environmental consciousness while purchasing product across different categories of respondents: Recycling save natural resources; Excessive packaging damages environment; Refuse to buy from accused retailer; Avoid buying from irresponsible company; Buying in refillable containers. 


\section{Conclusion and Suggestions:-}

The present study suggests that there are important differences in factors which affect various forms of socially responsible and environmentally conscious behaviors. The findings suggest that there is a viable and identifiable market segment with high purchase intensions concerning environmental friendly packaged products. To improve our understanding of the environmentally conscious consumer and the buying process, further research efforts should focus upon several other dimensions as well.

\section{References:-}

1. Autio, M. and Heininem, V. (2004), "To Consume or not to Consume?Young People's Environmentalism in the Affluent Finnish Society", Young, Nardic Journal of Youth Research, Vol. 12, Issue 2, 137-153.

2. Barr, S. (2007), "Factors Influencing Environmental Attitude and Behavior, A U.K. Case Study of Household Waste Management", Environment and Behavior, Vol. 39 Issue 4, 435-472.

3. Ellen, P.S., Wiener, J.L. and Walgren, C.C. (1991), "The Role of Perceived Consumer Effectiveness in Motivating Environmentally Conscious Behaviors", Journal of Public Policy and marketing, Vol. 10 (2), 102 117.

4. Krause, D. (1993), "Environmental Consciousness: An Empirical Study", Environment and Behavior, Vol. 25, Issue 1, 126-142.

5. Ropke, I. (2001b), “The Environmental Impact of Changing Consumption Patterns: A Survey”, International Journal of Environment and Pollution, Vol. 15, 127-145.

6. Zimmer, M.R., Stafford, T.F. and Stafford, M.R. (1994), "Green Issues: Dimensions of Environmental Concern", Journal of Business Research, Vol. 30 (1), 63-74. 